\title{
Mental disorders of known aetiology and precision medicine in psychiatry: a promising but neglected alliance
}

\author{
D. Fraguas $^{1 *}$, C. M. Díaz-Caneja ${ }^{1}$, M. W. State ${ }^{2}$, M. C. O’Donovan ${ }^{3}$, R. E. Gur ${ }^{4}$ and C. Arango ${ }^{1}$ \\ ${ }^{1}$ Department of Child and Adolescent Psychiatry, Hospital General Universitario Gregorio Marañón, CIBERSAM, IiSGM, School of Medicine, \\ Universidad Complutense de Madrid, Madrid, Spain \\ ${ }^{2}$ Department of Psychiatry and Langley Porter Psychiatric Institute, University of California, San Francisco, CA, USA \\ ${ }^{3}$ Department of Psychological Medicine and Clinical Neuroscience, MRC Centre for Neuropsychiatric Genetics and Genomics, Cardiff University \\ School of Medicine, Heath Park, Cardiff, UK \\ ${ }^{4}$ Department of Psychiatry, Neuropsychiatry Section, Perelman School of Medicine, University of Pennsylvania, Philadelphia, PA, USA
}

Personalized or precision medicine is predicated on the assumption that the average response to treatment is not necessarily representative of the response of each individual. A commitment to personalized medicine demands an effort to bring evidence-based medicine and personalized medicine closer together. The use of relatively homogeneous groups, defined using a priori criteria, may constitute a promising initial step for developing more accurate risk-prediction models with which to advance the development of personalized evidence-based medicine approaches to heterogeneous syndromes such as schizophrenia. However, this can lead to a paradoxical situation in the field of psychiatry. Since there has been a tendency to loosely define psychiatric disorders as ones without a known aetiology, the discovery of an aetiology for psychiatric syndromes (e.g. 22q11.2 deletion syndrome in some cases of schizophrenia), while offering a path toward more precise treatments, may also lead to their reclassification away from psychiatry. We contend that psychiatric disorders with a known aetiology should not be removed from the field of psychiatry. This knowledge should be used instead to guide treatment, inasmuch as psychotherapies, pharmacotherapies and other treatments can all be valid approaches to mental disorders. The translation of the personalized clinical approach inherent to psychiatry into evidence-based precision medicine can lead to the development of novel treatment options for mental disorders and improve outcomes.

Received 25 January 2016; Revised 5 May 2016; Accepted 5 May 2016; First published online 23 June 2016

Key words: Personalized medicine, psychiatry, risk-prediction models, schizophrenia, 22q11.2 deletion syndrome.

For a doctor seems not even to study health in this way [by studying the idea of health], but the health of man, or perhaps rather the health of a particular man; it is individuals the he is healing (Aristotle, Nicomachean Ethics, I, 6, 1097a)

Personalized medicine, now generally referred to as precision medicine, which can be described as providing the right patient with the right treatment at the right dose at the right time, has become a promising and controversial issue in healthcare research in recent years (Schork, 2015). Precision medicine proposes the use of an individual's genetic and epigenetic information, clinical symptomatology, observable biomarkers, and environmental factors to tailor the best therapeutic option to each person in each condition (Ozomaro et al. 2013) and, further, to implement the best preventive

* Address for correspondence: Dr D. Fraguas, Department of Child and Adolescent Psychiatry, Hospital General Universitario Gregorio Marañón, CIBERSAM, IiSGM, School of Medicine, Universidad Complutense de Madrid, Madrid, Spain

(Email: david.fraguas@cibersam.es) strategy for specific diseases or conditions in specific groups or communities (Gillman \& Hammond, 2016).

Psychiatric disorders, such as schizophrenia, depression, anxiety, obsessive-compulsive and autism spectrum disorders, as well as some disorders from other fields of medicine such as oncology and neurology, are heterogeneous syndromes rather than specific diseases (Paulus, 2015). Thus, they constitute important targets for personalized medicine. Therapeutic research in psychiatry has generally implicitly treated psychiatric disorders as homogenous conditions resulting from a defined, yet still largely undiscovered, set of aetiological factors. Yet there is overwhelming evidence that psychiatric categories might be best conceptualized as heterogeneous endpoints resulting from multiple aetiological processes, each of which in turn may be caused by a large number of genetic and environmental factors, some directly influencing brain structure and function (van Os et al. 2010), others possibly doing so indirectly, for example via the immune system.

Personalized or precision medicine assumes that the average response to treatment is not necessarily 
representative of the response of each individual. Indeed it may not even be representative for any individual (de Leon, 2012). If this hypothesis is applicable to psychiatry, it may help explain the failure to develop novel pharmacological treatments in psychiatry (Paulus, 2015) despite the advances that have been made in at least partially understanding some of the neurobiological bases of psychiatric disorders in recent years (Bargmann, 2015).

An alternative approach to optimize research on treatment of psychiatric disorders relies on riskprediction models. Such models, rooted in a Bayesian approach to uncertainty reasoning, encourage a pragmatic focus on prediction, with the aim of estimating the probability or risk of other precise outcomes within a specific period at an individual level. Therefore, they constitute valuable tools to guide the implementation of specific solutions for specific conditions, which is the basis of personalized medicine (Paulus, 2015).

Risk-prediction models are based on the identification and analysis of demographic, clinical, and biological markers that allow the formation of more homogeneous groups, up to the extreme scenario of $\mathrm{N}$-of-1 groups and N-of-1 trials (Schork, 2015), close to or in line with idiographic research approaches (Ng \& Weisz, 2016). In practice, this involves understanding everyday clinical care as a series of N-of-1 trials (Schork, 2015) or, more realistically, subdividing psychiatric syndromes into more homogeneous groups (Ng \& Weisz, 2016), thus reducing the inherent heterogeneity of psychiatric syndromes. This can be seen as an iterative approach, and incorporation of data obtained from these more homogenous groups may benefit further development of more accurate riskprediction models. This is because, while the approach of assessing heterogeneous syndromes as a single entity substantially reduces the effect size of predictors, the use of more homogeneous subgroups facilitates the identification of new predictors, thus leading to improved risk-prediction models (Wen \& Lu, 2013).

The problem of lack of homogeneity in clinical studies, especially in drug randomized control trials, has been a recurrent theme in recent decades (Feinstein \& Horwitz, 1997). As a result, the use of new statistical techniques that make it possible to describe patient populations as a whole and patients as individuals, such as random-effects linear models, has been encouraged (Schork, 2015). Unfortunately, these recommendations have still not been incorporated into most evidence-based studies (Schork, 2015). Schematically, evidence-based medicine approaches focus on average data to define one-size-fits-all treatments, whereas personalized or precision medicine aims to obtain customized treatments for each specific individual or group. In a sense, evidence-based medicine deliberately ignores outliers, while personalized medicine pays special attention to outliers (de Leon, 2012; Schork, 2015).

A commitment to personalized medicine demands a major effort to bring evidence-based medicine and personalized medicine closer together, in an attempt to achieve, insofar as possible, personalized evidencebased medicine (de Leon, 2012; Ng \& Weisz, 2016). Doing so may be hindered by factors such as practical difficulties in implementing personalized treatments for mental disorders, methodological difficulties in designing clinical trials with a personalized medicine approach, barriers to addressing public health issues, dualistic thinking regarding psychiatric illness (e.g. organic-functional/hardware-software dichotomies), unrealistic expectations about personalized medicine, conflicts of interest (Kendler, 2012) or potential measurement burden due to increase of clinical and/ or biological assessments. Moreover, the fact that the aetiology of most mental disorders is far from being clarified poses additional, non-negligible challenges.

For these reasons, relatively homogeneous groups defined using a priori criteria, such as common genetic, biological or environmental factors, may constitute the initial phases of developing more accurate models with which to advance the development of personalized evidence-based medicine approaches and eventually to advance the understanding of the biological underpinnings of psychiatric syndromes (de Leon, 2012).

The case of schizophrenia, as a highly heterogeneous psychiatric syndrome, may be useful to illustrate this issue. The heterogeneity of schizophrenia has been confirmed in various domains, including genetics, neurodevelopment, neuroanatomy, symptoms, treatment response, and outcomes (van Os et al. 2010). The attempt to tackle such a heterogeneous disorder with the very narrow range of different therapeutic alternatives that are partially effective on some of the core symptoms of the disease (i.e. positive symptoms) but have close to no effect on others (i.e. negative and cognitive symptoms) could explain, to some extent, why there has been little improvement in functional outcome in the past century (Hegarty et al. 1994). For decades, authors from different theoretical schools have called for the use of more homogeneous categories to guide the treatment of schizophrenia. However, attempts to assess treatment response according to more homogenous clinical subgroups in schizophrenia have been scarce (Kirkpatrick et al. 2000; de Leon, 2014), and have been only limitedly tested in randomized clinical trials (Umbricht et al. 2014).

In this scenario, there is a need for novel methodological strategies that can help develop new treatment options. Risk-prediction models may offer a unique 
opportunity to assess the effectiveness of new or even not so new treatment options in a personalizedmedicine (or proxy) framework. Unfortunately, current knowledge is not sufficiently developed to allow for the production of accurate risk-prediction models applicable to precision medicine at a clinical level.

A multiclass likelihood-ratio approach, based on a forward selection algorithm that considers high-order and high-dimensional gene-gene/gene-environment interactions, could be used to define homogeneous groups and improve the accuracy of these potential risk-prediction models (Wen \& Lu, 2013). However, implementation of this approach for the study of schizophrenia may well be hindered by the extreme complexity and multidimensional nature of this condition, which undermines the validity and reliability of these models.

An alternative approach is the use of biological mechanism-based subgroups based on shared specific underlying neural processes or aetiological factors. As an example, we take chromosome 22q11.2 deletion syndrome (22q11.2DS). This syndrome, also called velocardiofacial syndrome or DiGeorge syndrome, is associated with high rates of schizophrenia spectrum (up to $40 \%$ in adults) and other psychiatric disorders [e.g. mood and anxiety disorders, obsessive-compulsive disorder (OCD)]. It is usually defined as a genomic neurodevelopmental disorder with markedly variable expressivity in clinical and cognitive presentation. Remarkably, there is no specific symptomatic profile in subjects with 22q11.2DS schizophrenia, which instead encompasses the range of the disorder in the general schizophrenia population (Owen \& Doherty, 2016).

Nevertheless, among subjects with schizophrenia, those who also have 22q11.2DS must constitute a relatively homogenous group in which shared aetiological factors presumably lead to more similar pathophysiology compared with random pairs of individuals drawn from the whole population of people with the disorder. Prevalence of 22q11DS in patients with schizophrenia is estimated at about $0.30 \%$ (Rees et al. 2014). Models based on 22q11.2DS would therefore have a direct impact on only a minority of subjects with schizophrenia. Still, they provide an appropriate scenario for developing better risk-prediction models and for testing specific responses to specific treatment options. This is all the more applicable as recent results suggest that neurons derived from induced pluripotent stem cells (iPSCs) could constitute a valid in vitro model of schizophrenia in 22q11.2DS, since they recapitulate the microRNA expression patterns expected of in vivo 22q11.2 haploinsufficiency (Zhao et al. 2015), thus broadening the opportunities to identify new targets for intervention.
This is not to say that 22q11.2DS defines a precisely aetiologically homogeneous clinical subgroup of schizophrenia. In fact, clinical expression in subjects with 22q11.2DS and other copy number variations $(\mathrm{CNV})$ is, as noted, highly variable, likely a consequence to some degree of contributing common alleles across the genome (Tansey et al. 2015) (i.e. 'genetic background') as well as the variable contribution of multiple small effect genes within the $\mathrm{CNV}$ region (Sanders et al. 2015). Recent promising research on treatment strategies in autism spectrum disorder phenotypes focusing on known genetic aetiologies, such as fragile $X$ syndrome (Hagerman et al. 2014) or Phelan-McDermid syndrome (Harony-Nicolas et al. 2015), are two other valuable examples of using biological mechanism-based subgroups for the development of precise treatments.

In addition to the identification of more homogeneous populations based on genetic factors, personalized medicine efforts in psychiatry could benefit from subtyping based on other biological variables such as error-related negativity in OCD, and from novel computer techniques allowing for the collection and interpretation of large amounts of real-time data at the individual level, such as ecology momentary assessments (Veling et al. 2016). This could help identify triggers and early signs of psychopathology for each individual and favour the development of personalized risk-prediction algorithms for patients with depression, anxiety, psychosis and other mental disorders.

Perhaps uniquely in medicine, psychiatry faces a paradoxical situation that, unless guarded against, poses a potential hindrance to the development of personalized treatments for mental disorders. Historically, there has been a tendency to loosely define psychiatric disorders as ones without a known or observable aetiology, and the discovery of an aetiology has tended to lead to a reclassification away from psychiatric toward medical conditions and to transfer of care outside psychiatry (Arango \& Fraguas, 2016). While a common aetiological factor makes schizophrenia in patients with 22q11DS a more homogeneous condition, whose study has the potential to help personalized medicine move forward in the field of psychiatry, knowledge of this aetiological factor puts 22q11.2DS schizophrenia at risk of being reclassified as a 'medical' disease. Thus, while discovery of the aetiology of psychiatric syndromes offers a path toward both more precise treatments and a deeper general understanding of pathophysiological mechanisms, psychiatry may lose its role of caring for people with these conditions (Arango \& Fraguas, 2016).

Many medical conditions have a relatively wellknown pathophysiology (e.g. diabetes) or an 
immediate aetiology (e.g. stroke) but an underlying aetiology that remains undiscovered. However, most psychiatric disorders have not only an unidentified underlying aetiology but also an unknown proximate cause and pathophysiology. Within this framework, should schizophrenia related to 22q11.2DS or any other psychiatric disorder with a known aetiology (or supposedly known aetiology) be removed from the realm of psychiatry just because of a known medical aetiology? We suggest the answer should be a resounding no. To argue otherwise is implicitly dualistic and conjures the ghost of Descartes. Moreover, even in the hypothetical and improbable event that we were to discover that all mental disorders have a specific, albeit complex, biological aetiology, in the absence of a simple cure, there will inevitably still be a range of environmental factors (and gene-environment interactions) to be dealt with in clinical practice, and their multiple symptomatic, cognitive, and/or behavioural manifestations require psychiatric approaches, both medical and non-medical. In the current context, we note that doing so is also likely to hamper the development of personalized medicine in psychiatry. On the contrary, conditions such as schizophrenia related to 22q11.2DS should serve as opportunities for psychiatry to become a more collaborative and multidisciplinary specialty.

Psychiatrists deal with unique subjects with unique sets of factors that drive their achievements and difficulties. At the end of the day, every clinical consultation needs a personalized medicine approach. As clinicians, it is also our responsibility to contribute to the development of better and, insofar as possible, more precise treatments. Psychotherapies, pharmacotherapies or other treatments can be all valid approaches to complex brain conditions such as mental disorders. As one among several clinical neuroscience disciplines including neurology and neurosurgery, psychiatry will require training in converging diagnostic methods and therapeutic interventions. This could help psychiatry distance itself from previous dogmatic or quasi-dogmatic approaches that have conditioned the development of the specialty over time. Otherwise, psychiatrists will lack the practical abilities required to manage mental disorders in an efficient manner.

Research efforts to translate the personalized clinical approach inherent to psychiatry into evidence-based precision medicine can pave the way to success in the search for optimal treatment options for mental disorders.

\section{Acknowledgements}

Supported by the Spanish Ministry of Economy and Competitiveness. Instituto de Salud Carlos III,
CIBERSAM. Madrid Regional Government (S2010/ BMD-2422 AGES), European Union Structural Funds and European Union Seventh Framework Programme under grant agreements FP7-HEALTH2009-2.2.1-2-241909 (Project EU-GEI), FP7-HEALTH2009-2.2.1-3-242114 (Project OPTiMISE), FP7- HEALTH2013-2.2.1-2-603196 (Project PSYSCAN) and FP7HEALTH-2013-2.2.1-2-602478 (Project METSY); Fundación Alicia Koplowitz and Fundación Mutua Madrileña, and ERA-NET NEURON (Network of European Funding for Neuroscience Research).

\section{Declaration of Interest}

David Fraguas has been a consultant and/or advisor to or has received honoraria from AstraZeneca, BristolMyers Squibb, Janssen, Lundbeck, Otsuka, and Pfizer. Covadonga M. Díaz-Caneja has previously held a Río Hortega grant, Instituto de Salud Carlos III, Spanish Ministry of Economy and Competitiveness, and a grant from Fundación Alicia Koplowitz. Matthew W. State serves on scientific advisory boards for Pfizer Pharmaceuticals, Blackthorn Therapeutics and SynapDx. Michael C. O'Donovan has received a consultancy fee from Roche in return for advice on the implications of current genetic findings in psychiatry. Celso Arango has been a consultant to or has received honoraria or grants from Abbott, Amgen, AstraZeneca, Bristol-Myers Squibb, Caja Navarra, CIBERSAM, Fundación Alicia Koplowitz, Instituto de Salud Carlos III, Janssen-Cilag, Lundbeck, Merck, Ministerio de Ciencia e Innovación, Ministerio de Sanidad, Ministerio de Economía y Competitividad, Mutua Madrileña, Otsuka, Pfizer, Roche, Servier, Shire, Takeda, and Schering-Plough. Raquel E. Gur has no conflict of interest to declare.

\section{References}

Arango C, Fraguas D (2016). Should psychiatry deal only with mental disorders without an identified medical aetiology? World Psychiatry 15, 22-23.

Bargmann CI (2015). How the new neuroscience will advance medicine. Journal of the American Medical Association 314, 221-222.

De Leon J (2012). Evidence-based medicine versus personalized medicine: are they enemies? Journal of Clinical Psychopharmacology 32, 153-164.

De Leon J (2014). Focusing on drug versus disease mechanisms and on clinical subgrouping to advance personalised medicine in psychiatry. Acta Neuropsychiatrica 26, 327-333.

Feinstein AR, Horwitz RI (1997). Problems in the 'evidence' of 'evidence-based medicine'. American Journal of Medicine 103, 529-535. 
Gillman MW, Hammond RA (2016). Precision treatment and precision prevention: integrating 'below and above the skin'. JAMA Pediatrics 170, 9-10.

Hagerman RJ, Des-Portes V, Gasparini F, Jacquemont S, Gomez-Mancilla B (2014). Translating molecular advances in fragile $\mathrm{X}$ syndrome into therapy: a review. Journal of Clinical Psychiatry 75, e294-e307.

Harony-Nicolas H, De Rubeis S, Kolevzon A, Buxbaum JD (2015). Phelan Mcdermid syndrome: from genetic discoveries to animal models and treatment. Journal of Child Neurology 30, 1861-1870.

Hegarty JD, Baldessarini RJ, Tohen M, Waternaux C, Oepen G (1994). One hundred years of schizophrenia: a metaanalysis of the outcome literature. American Journal of Psychiatry 151, 1409-1416.

Kendler KS (2012). The dappled nature of causes of psychiatric illness: replacing the organic-functional/ hardware-software dichotomy with empirically based pluralism. Molecular Psychiatry 17, 377-388.

Kirkpatrick B, Kopelowicz A, Buchanan RW, Carpenter Jr. WT (2000). Assessing the efficacy of treatments for the deficit syndrome of schizophrenia. Neuropsychopharmacology 22, 303-310.

Ng MY, Weisz JR (2016). Annual research review: building a science of personalized intervention for youth mental health. Journal of Child Psychology and Psychiatry 57, 216-236.

Owen MJ, Doherty JL (2016). What can we learn from the high rates of schizophrenia in people with 22q11.2 deletion syndrome? World Psychiatry 15, 23-25.

Ozomaro U, Wahlestedt C, Nemeroff CB (2013). Personalized medicine in psychiatry: problems and promises. BMC Medicine 11, 132.

Paulus MP (2015). Pragmatism instead of mechanism: a Call for impactful biological psychiatry. JAMA Psychiatry 72, 631-632.

Rees E, Walters JT, Georgieva L, Isles AR, Chambert KD, Richards AL, Mahoney-Davies G, Legge SE, Moran JL, Mccarroll SA, O'donovan MC, Owen MJ, Kirov G (2014). Analysis of copy number variations at 15 schizophrenia-associated loci. British Journal of Psychiatry 204, 108-114.
Sanders SJ, He X, Willsey AJ, Ercan-Sencicek AG, Samocha KE, Cicek AE, Murtha MT, Bal VH, Bishop SL, Dong S, Goldberg AP, Jinlu C, Keaney 3rd JF, Klei L, Mandell JD, Moreno-De-Luca D, Poultney CS, Robinson EB, Smith L, Solli-Nowlan T, Su MY, Teran NA, Walker MF, Werling DM, Beaudet AL, Cantor RM, Fombonne E, Geschwind DH, Grice DE, Lord C, Lowe JK, Mane SM, Martin DM, Morrow EM, Talkowski ME, Sutcliffe JS, Walsh CA, Yu TW, Ledbetter DH, Martin CL, Cook EH, Buxbaum JD, Daly MJ, Devlin B, Roeder K, State MW (2015). Insights into autism spectrum disorder genomic architecture and biology from 71 risk loci. Neuron 87, 1215-1223.

Schork NJ (2015). Personalized medicine: time for one-person trials. Nature 520, 609-611.

Tansey KE, Rees E, Linden DE, Ripke S, Chambert KD, Moran JL, McCarroll SA, Holmans P, Kirov G, Walters J, Owen MJ, O'donovan MC (2015). Common alleles contribute to schizophrenia in CNV carriers. Molecular Psychiatry. Published online: 22 September 2015. doi:10.1038/mp.2015.143.

Umbricht D, Alberati D, Martin-Facklam M, Borroni E, Youssef EA, Ostland M, Wallace TL, Knoflach F, Dorflinger E, Wettstein JG, Bausch A, Garibaldi G, Santarelli L (2014). Effect of bitopertin, a glycine reuptake inhibitor, on negative symptoms of schizophrenia: a randomized, double-blind, proof-of-concept study. JAMA Psychiatry 71, 637-646.

Van Os J, Kenis G, Rutten BP (2010). The environment and schizophrenia. Nature 468, 203-212.

Veling W, Pot-Kolder R, Counotte J, Van Os J, Van Der Gaag M (2016). Environmental social stress, paranoia and psychosis liability: a virtual reality study. Schizophrenia Bulletin. Published online: 2 April 2016. doi:10.1093/schbul/sbw031.

Wen Y, Lu Q (2013). A multiclass likelihood ratio approach for genetic risk prediction allowing for phenotypic heterogeneity. Genetic Epidemiology 37, 715-725.

Zhao D, Lin M, Chen J, Pedrosa E, Hrabovsky A, Fourcade HM, Zheng D, Lachman HM (2015). MicroRNA profiling of neurons generated using induced pluripotent stem cells derived from patients with schizophrenia and schizoaffective disorder, and 22q11.2 Del. PLOS ONE 10, e0132387. 